\title{
广义 $\boldsymbol{J}$ - 同 态 \\ 李贵松 \\ (中国科学院系统科学研究所, 北京 100080)
}

关锤词广义 $J$-同态、实和复投影空问上的向量丛、Rohlin 定理

\section{一、引 言}

设 $\phi \in \widetilde{K O}(X)$, 其中 $X$ 为紧致拓扑空间. 以 $\Omega_{n}(X, \phi)$ 记 $X$ 的 $\phi$ 为系数的第 $n$ 个法协 边群. 假设 $X$ 为道路连通, 则由自然同态 $I(\phi): \pi_{3}^{3} \cong \Omega_{3}(*, 0) \rightarrow Q_{3}(X, \phi)$ 及经典的稳定 $J$-同态, 我们可以唯一地定义一个所谓的广义 $J$-同态 $J(\phi): \pi_{3}(S O) \rightarrow \Omega_{3}(X, \phi)$. 我们以 $i(\phi)$ 来记有限循环群 $\operatorname{Im} J(\phi)$ 的阶, 有关细节请参见文献 [1].

本文中利用 Gysin 序列及 Koschorke 序列 ${ }^{[2]}$, 对实或复投影空间上的向量丛 $\phi$ 决定了 $j(\phi)$. 作为应用我们还给出了 Habegger ${ }^{[3]}$ 的结果的一个新证明.

\section{二、主 要 结 果}

我们首先证明如下的

定理 1 令 $\eta$ 为 $\mathrm{C} P^{n}$ 上的典则复线丛, $[$,$] 表示最大公因子. 若 n \geqslant 2$, 则对任意 $k \in \mathbf{Z}$ 均有 1) $J(k \eta)$ 为满同态；2) $i(k \eta)=[24,|k|]$.

证 对于球丛 $p: S(\eta)=S^{2 n+1} \rightarrow \mathbb{C} P^{n}$ 我们有交换图

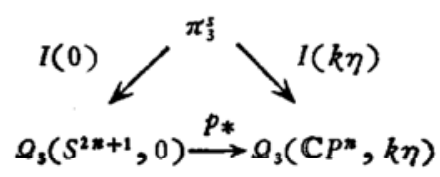

因 $n \geqslant 2$, 有 $I(0)$ 为同构, 于是 $\operatorname{Im} J(k \eta)=\operatorname{Im} p_{*}$. 由文献 [2] 中命题 9.20 , 相应于球丛 $p: S^{2 n+1} \rightarrow \mathrm{C} P^{n}$ 有如下的 Gysin 正合序列:

$$
\begin{aligned}
& \Omega_{3}\left(S^{2 n+1}, 0\right) \stackrel{P_{*}}{\longrightarrow} \Omega_{3}\left(\mathrm{C} P^{n}, k \eta\right) \rightarrow \Omega_{1}\left(\mathrm{C}^{n},(k+1) \eta\right) \rightarrow \\
& \Omega_{2}\left(S^{2 n+1}, 0\right) \rightarrow \Omega_{2}\left(\mathrm{C} P^{n}, k \eta\right) \rightarrow
\end{aligned}
$$

对 $n \geqslant 1$ 由文献 [4] 中的结果可知, 视 $k$ 为奇或偶, $Q_{1}\left(\mathbf{C} P^{n}, k \eta\right)$ 分别同构于 0 及 $\mathbb{Z}_{2}$, 而 $\boldsymbol{Q}_{2}\left(\mathrm{C} \boldsymbol{P}^{n}, k \eta\right)$ 则分别同构于 0 及 $\mathrm{Z} \oplus \mathrm{Z}_{2 \text { 。 }}$ 于于是针对 $k$ 为奇或偶两种情形, 上述正合序列依次 取下面两个形式:

$$
\begin{aligned}
& \mathbb{Z}_{24} \stackrel{p_{*}}{\longrightarrow} \Omega_{3}\left(\mathbf{C} P^{n}, k \eta\right) \rightarrow \mathbf{Z}_{2} \rightarrow \mathbf{Z}_{2} \rightarrow 0 ; \\
& \mathbf{Z}_{24} \stackrel{P_{*}}{\longrightarrow} \Omega_{3}\left(\mathbf{C} P^{n}, k \eta\right) \rightarrow 0 .
\end{aligned}
$$

本文 1989 年 7 月 31 日收到. 
由此可知 $p_{*}$ 恒为满同态. 论断 1) 得证. 设 $k$ 为偶数, 则

2) 在文献 [1] 中已得到证明。在此仅考虑 $k$ 为奇数的情形. 由 1) 只需计算 $Q_{3}(\mathrm{CP} *$; $k \eta)$. 由文献 [4] 我们对奇数 $k$ 有

$$
\Omega_{2}\left(\mathrm{C} P^{n} \times B O(2), k \eta+\Gamma\right) \cong \mathbf{Z} \oplus \mathbf{Z}_{4},
$$

于是我们有如下的 Koschorke 序列(见文献 [2] 定理 9.3):

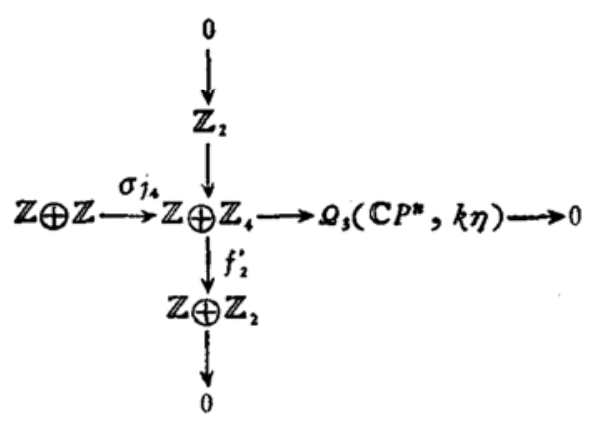

其中 $\mathrm{Z} \oplus \mathrm{Z}$ 由 $A=\left[\mathrm{C} P^{2}\right.$, 常值映射, or $]$ 及 $B=\left[C P^{2}\right.$, 含入映射, or $]$ 生成. 不难算出

$$
\begin{aligned}
& f_{2}^{\prime} \sigma j_{4}(A)=( \pm 3,1), \\
& f_{2}^{\prime} \sigma j_{4}(B)=( \pm 3 \pm k, ?),
\end{aligned}
$$

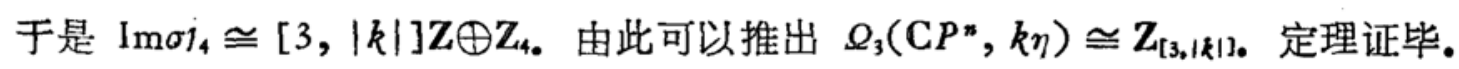

注 1 在定理 1 中若令 $n=1$, 则由 Koschorke 序列及文献 [1] 中的方法可以证明 $i(k \eta)=24$ 或 3 分别视 $k \in \mathbf{Z}$ 为偶或奇而定.

现在考虑实投影空间的情形.

引理 1 设 $\xi$ 为 $\mathrm{R} P^{n}$ 上的典则实线丛, $n \geqslant 4$. 对 $k \in \mathrm{Z}$ 群 $\Omega_{m}\left(\mathrm{R} P^{n}, k \xi\right)$ 可由下表 给出:

\begin{tabular}{c|c|c|c} 
& $m=1$ & $m=2$ & $m=3$ \\
\hline$k \equiv 0(4)$ & $\mathbb{Z}_{2} \oplus \mathbb{Z}_{2}$ & $\mathbb{Z}_{2} \oplus \mathbb{Z}_{2}$ & $?$ \\
\hline$k \equiv 1(4)$ & $\mathbb{Z}_{2}$ & $\mathbb{Z}_{8}$ \\
\hline$k \equiv 2(4)$ & $\mathbb{Z}_{4}$ & 0 & $\mathbb{Z}_{12}$ \\
\hline$k \equiv 3(8)$ & 0 & $\mathbb{Z}_{2}$ & $\mathbb{Z}_{2} \oplus \mathbb{Z}_{2}$ \\
\hline$k \equiv 7(8)$ & $\mathbb{Z}_{2}$ & $\mathbb{Z}_{2}$ \\
\hline
\end{tabular}

证设 $k \geqslant m+2$ 则由文献 [2] 中命题 5.4 知有同构

$$
\Omega_{m}\left(\mathbb{R} P^{n}, k \xi\right) \cong \pi_{m+n}\left(V_{k+n+1, n+1}\right),
$$

若 $k \geqslant 5$ 则引理由文献 [5] 中关于 Stiefel 流形的同伦群的结果可证. 令 $\rho(n)$ 表集合 $\{0<s \leqslant n \mid s \equiv 0,1,2,4(8)\}$ 的元素个数. 则在 $\widetilde{K O}\left(\mathbf{R} P^{n}\right)$ 中有 $2^{\rho(n)} \xi=0$. 注意对 $n \geqslant 4$ 我们有 $2^{\rho(n)} \equiv 0(8)$, 而 $\Omega_{m}(\mathbb{R} P n, k \xi)$ 仅依赖于 $k \xi$ 在 $\widetilde{K O}$ 中的类. 于是一般情形可归 结为如上的特殊情形.

考虑二重复迭 $p: S(\xi)=S^{n} \rightarrow \mathbb{R} P^{n}$. 类似于定理 1 的证明可知 $(n \geqslant 4)$ :

$$
\operatorname{Im} J(k \xi)=\operatorname{Im} p_{*}: \Omega_{3}\left(S^{n}, 0\right) \rightarrow \Omega_{3}\left(\mathbb{R} P^{n}, k \xi\right) .
$$

对上述二重复迭我们亦有 Gysin 序列(见文献 [2] 命题 9.20)

$$
\Omega_{3}\left(S^{n}, 0\right) \stackrel{P_{*}}{\longrightarrow} \Omega_{3}\left(\mathbb{R} P^{n}, k \xi\right) \rightarrow \Omega_{2}\left(R P^{n},(k+1) \xi\right) \rightarrow
$$

第 21 期 


$$
Q_{2}\left(S^{n}, 0\right) \rightarrow \Omega_{2}\left(\mathrm{R} P^{n}, k \xi\right) \rightarrow \Omega_{1}\left(\mathrm{R} P^{n},(k+1) \xi\right) \rightarrow
$$

利用引理 1 及对上面正合序列的分析可以证明如下的 $(k \equiv 0(4)$ 的情形, 见文献 [1])

定理 2 设 $\xi$ 为 $\mathbf{R} P^{n}$ 上的典则实线丛, $n \geqslant 4$. 则对 $k \in \mathbf{Z}$ 阶 $j(k \xi)$ 由下表给出:

\begin{tabular}{c|c|c|c|c|c|}
\hline$k$ & $0(4)$ & $1(4)$ & $2(4)$ & $3(8)$ & $7(8)$ \\
\hline$j(k \xi)$ & 24 & 2 & 12 & 2 & 1 \\
\hline
\end{tabular}

注 2 由阻碍论可知若 $X$ 为 3 -连通, 则对任意 $\phi \in \widetilde{K O}(X)$ 均有 $j(\phi)=24$.

注 3 设 $\alpha$ 为 $\pi_{3}(S O) \cong \mathrm{Z}$ 的生成元. 则 $\alpha$ 可视为流形 $X$ (维数 $\geqslant 4$ )中的 3 维标架式浸 人子流形. 由文献 [1] 中的讨论可知 $i(-T X)$ 恰为 $\alpha$ 在标架协边群 $\Lambda_{3}(X) \cong \Omega_{3}(X,-T X)$ 中的阶. 于是定理 1 和 2 事实上给出了 $\alpha$ 作为投影空间中的标架式浸人子流形在相应的标架 协边群中的阶。

\section{三、关于 Habegger 的一个结果}

著名的 Rohlin 定理指出: 设 $M$ 为具有偶相交形式的单连通 4 流形则 $M$ 的指标（signature) $\sigma(M)$ 模 16 同余于 0 . 这一结果对非单连通流形通常是不正确的. 也就是说有

命题 存在具有偶相交形式的 4 维定向流形 $M$ 使得 $\sigma(M)=8$.

Habegger 在文献 [3] 中通过构造具体例子证明了这一命题. 在此我们将给出该命题的 一个非构造性的证明. 首先我们有

\section{引理 2 存在定向的 4 流形 $M$ 满足}

1) $w_{2}(M)=x^{2}$, 其中 $x \in H^{1}\left(M, Z_{2}\right)$ :

2) $\sigma(M)=8$.

证令 $\circ$ 为满足 1) 的全体定向 4 流形 $M$ 的 Pontrjagin 数 $p_{1}(M)[M]=3 \sigma(M)$ 的最 大公因子, 则由定理 2 及文献 [1] 中命题 4 可知 $c=24$. 引理得证.

我们以 $\rho$ 记模 2 同态, 以 $\delta$ 记关联于短正合序列

$$
0 \rightarrow \mathrm{Z} \stackrel{\times 2}{\longrightarrow} \mathrm{Z} \rightarrow \mathrm{Z}_{2} \rightarrow 0
$$

的 Bockstein 上边缘同态, 则有 $s_{q^{1}}=\rho \delta$. 设 $M$ 满足引理 2 中的 1) 则有 $w_{2}(M)=s_{q^{1}}(x)=$ $\rho(\delta(x)) \in \rho\left(H^{2}(M, Z)\right.$ 的挠子群). 于是 $M$ 具有偶相交形式. 命题成为引理 2 的一个推论.

致谢：作者对导师李邦河研究员的鼓励和邦助表示感谢.

\section{参考文献}

[1] 李贯松,科学通报, 34(1989), 16: 1206-1208.

[2] Koschorke, U., Lecture Notes in Math. 847, Springer, 1981.

[3] Habegger, N., Comment. Math. Helvetici, 57(1982),22-24.

[4] 李邦河, 论 Koschorke 法协边序列(待发表).

[5] Paechter, G., Qaurt. J. Math. Oxford, 7(1956), 249-268. 